R. S. Manni

Nagoya Math. J.

Vol. 118 (1990), 165-176

\title{
PROJECTIVE VARIETIES AND RINGS OF THETANULLWERTE
}

\author{
RICCARDO SALVATI MANNI
}

\section{Introduction}

Let $r$ denote an even positive integer, $m$ an element of $\boldsymbol{Q}^{2 g}$ such that $r \cdot m \equiv 0 \bmod 1$ and $\vartheta_{m}$ the holomorphic function on the Siegel upper-half space $\boldsymbol{H}_{g}$ defined by

$$
\vartheta_{m}(\tau)=\sum_{p \in \mathbb{Z}^{g}} \mathbf{e}\left((1 / 2)^{t}\left(p+m^{\prime}\right) \tau\left(p+m^{\prime}\right)+{ }^{t}\left(p+m^{\prime}\right) m^{\prime \prime}\right),
$$

in which $\mathbf{e}(t)$ stands for $\exp (2 \pi \sqrt{-1} t)$ and $m^{\prime}$ and $m^{\prime \prime}$ are the first and the second entry vector of $m$. Let $\Theta_{g}(r)$ denote the graded ring generated over $C$ by such Thetanullwerte; then it is a well known fact that the integral closure of $\Theta_{g}(r)$ is the ring of all modular forms relative to Igusa's congruence subgroup $\Gamma_{g}\left(r^{2}, 2 r^{2}\right)$ cf. [6]. We shall denote this ring by $A\left(\Gamma_{g}\left(r^{2}, 2 r^{2}\right)\right)$.

At the present the following results are known

$$
\Theta_{g}(r)=A\left(\Gamma_{g}\left(r^{2}, 2 r^{2}\right)\right)
$$

if and only if $g=1,2$ and $r=2 \mathrm{cf}$. [7], [8], [10]. In particular we have that $\Theta_{3}(2) \subsetneq A\left(\Gamma_{3}(4.8)\right)$.

Despite of this fact in [13] it has been proved that

$$
A\left(\Gamma_{3}\right) \subseteq \Theta_{3}(2)
$$

In view of these results we try to investigate this inclusion in higher genus, but we found that for any $g \geq 5$ certain Eisenstein series are not expressible as polynomials in the Thetanullwerte in $\Theta_{2}(2)$ cf. [11].

We have to recall another results of [8]. Let $\alpha$ be the morphism between Proj $A\left(\Gamma_{g}\left(r^{2}, 2 r^{2}\right)\right)$ and Proj $\Theta_{g}(r)$; then $\alpha$ is bijective if and only if $r=2$. Moreover $\alpha$ is not an isomorphism if $g \geq 6$. Let $\Gamma_{g}$ denote the full modular group and $\Gamma_{g}(2)$ the main congruence subgroup; then we put $B_{g}(2)=\Theta_{g}(2) \cap A\left(\Gamma_{g}(2)\right), B_{g}=B_{g}(2) \cap A\left(\Gamma_{g}\right)$. Clearly from a geometric

Received March 14, 1989. 
point of view the projective varieties associated to these two rings are very important and it would be very nice if the bijective morphisms were isomorphisms.

But the result that we shall obtain in the first part of this paper will contradict this. More precisely we shall get

$$
\alpha_{2}: \operatorname{Proj} A\left(\Gamma_{g}(2)\right) \longrightarrow \operatorname{Proj} B_{g}(2)
$$

is not an isomorphism if $g \geq 22$ and

$$
\alpha_{1}: \text { Proj } A\left(\Gamma_{g}\right) \longrightarrow \operatorname{Proj} B_{g}
$$

is not an isomorphism if $g \geq 47$.

In particular we have that there exists a modular form of arbitrarily high weight relative to $\Gamma_{g}$ or relative to $\Gamma_{g}(2)$ that is not in $\Theta_{g}(2)$. This gives a negative answer to our final consideration in [12]. Let $n$ be a positive integer we denote by $C_{g}(n)$ the graded ring generated over $C$ by all polynomials in the Thetanullwerte $\vartheta_{m}$, such that $2 n^{k} \cdot m \equiv 0 \bmod 1$ for some $k$ positive and integral, that are modular forms relative to $\Gamma_{g}$; then in the last section we shall prove that the projective variety associated to $C_{g}(n)$ is isomorphic to Proj $A\left(\Gamma_{g}\right)$, more precisely we prove that any modular form relative to $\Gamma_{g}$ of even weight $k$ with $k>2 g$ is in $C_{g}(n)$.

We wish to thank Prof. E. Freitag and Dr. S. Böcherer for helpful and friendly discussions about these topics.

$\S 1$.

In this section we shall recall some facts about lattices. For details we refer to [3]. First let us recall the definition of laminated lattice $\Lambda_{n}$. Let $\Lambda_{0}$ be the one-point lattice. For $n \geq 1$ we take all $n$-dimensional lattices with minimal norm 4 that have at least one sublattice $\Lambda_{n-1}$, and select those of minimal determinant. All $\Lambda_{n}$ for $n \leq 25$ are known and they are integral lattices if and only if $n \leq 24 . \quad \Lambda_{24}$ is the Leech lattice. Also in [3] p. 180 we have the following values for the determinants of $\Lambda_{21}, \Lambda_{22}, \Lambda_{23}, \Lambda_{24}: 32,12,4,1$.

In general by a $k$-dimensional section of a lattice $L_{n} \subseteq \boldsymbol{R}^{n}$ we mean a $k$-dimensional lattice $M_{k} \subseteq \boldsymbol{R}^{k} \subseteq \boldsymbol{R}^{n}$ such that $M_{k}=L_{n} \cap \boldsymbol{R}^{k}$. With these notations let us recall the following.

Proposition 1.

(i) Each of $\Lambda_{0} \cdots, \Lambda_{8}, \Lambda_{16}, \cdots, \Lambda_{23}$ has the smallest determinant of any 
section of the Leech lattice in its dimensions;

(ii) For $n \leq 23, \Lambda_{n}$ has the smallest determinant of any $n$ dimensional section of $\Lambda_{n+1}$.

A proof of this proposition can be found in [3].

A similar statement holds for the 48-dimensional integral lattice $P_{48 p}$ that has minimal norm 6 .

Proposition 2. In dimension $48-n$ the lattice $P_{48 p}$ has a section of determinant $d=3^{n} D$ where $D$ is given by the following table:

$$
\begin{array}{llllllll}
n=0 & 1 & 2 & 3 & 4 & 5 & 6 & 7 \\
D=1 & 2 & 3 & 4 & 4 & 4 & 4 & 4
\end{array}
$$

Moreover in dimensions 43 and above, these lattice have the smallest possible determinant of any section of any even unimodular 48-dimensional lattice with minimal norm 6.

Even in this case for details we refer to [3].

Let $L_{21}, L_{22}$ denote the matrices associated to the laminated lattices $\Lambda_{21}$ and $\Lambda_{22}$ with respect to a fixed basis, and $P_{47}, P_{46}$ the matrices associated to the 47 th and 46 th dimensional section of the lattice $P_{48 p}$ having the above mentioned properties; then with these notations we have the following

LEMMA 1.

a) There is no $\xi \neq 0$ in $Z^{22}$ such that $2 L_{22}-\xi^{t} \xi \geq 0$.

b) There is no $\xi \neq 0$ in $Z^{47}$ such that $4 P_{47}-\xi^{t} \xi \geq 0$.

Remark. Before proving the lemma we have mention that the similar statement for $E-\xi^{t} \xi \geq 0$ has been proved by Igusa in [8], using the matrix of Cartan integers associated with the Dynkin diagram of type $E_{6}$.

Moreover even the proof of the two above statemente is similar to that of [8].

Proof. In view of the remark we shall prove only the first statement. Assume that such a $\xi$ exists and is primitive. Since $G L(22, Z)$ acts transitively on the set of primitive vectors, there exists an integral invertible matrix $A$ such that $\xi={ }^{t} A^{-1} e_{1}$. In general we well denote by $e_{1}$ the $i$-th unit vector in $\boldsymbol{R}^{g}$. Therefore we have 


$$
{ }^{t} A L_{22} A-e_{1}{ }^{t} e_{1} \geq 0 \text {. }
$$

Let $B$ denote the square matrix of degree 21 obtained by crossing out the first row and the first column to ${ }^{t} A L_{22} A$, then we have

$$
\operatorname{det}\left(2^{t} A L_{22} A-e_{1}{ }^{t} e_{1}\right) \geq 2^{22} 12-2^{21} \operatorname{det} B \geq 0 \text {. }
$$

This implies $\operatorname{det} B \leq 24$, but this and $\operatorname{det} B \geq \operatorname{det} L_{21}=32$ bring a contradiction.

\section{$\S 2$.}

Let $\boldsymbol{H}_{g}$ denote the Siegel upper half-space, i.e. the set of complex $g$ by $g$ matrices $\tau$ such that $\operatorname{Im} \tau$ is positive definite, and $\operatorname{Sp}(g, R)$ the symplectic group with real coefficients. We know that the group of complex analytic automorphisms of $\boldsymbol{H}_{g}$ is given by $S p(g, \boldsymbol{R}) / \pm 1_{2 g}$ and if

$$
\sigma=\left[\begin{array}{ll}
a & b \\
c & d
\end{array}\right]
$$

then the complex analytic automorphism determinated by $\pm \sigma$ is

$$
\sigma \cdot \tau=(a \tau+b)(c \tau+d)^{-1} .
$$

Let $\Gamma_{g}$ denote the integral symplectic group. If $l$ is a positive integer we shall denote by $\Gamma_{g}(l, 2 l)$ the subgroup of $\Gamma_{g}$ defined by $\sigma \equiv 1_{2 g} \bmod l$ and $\operatorname{diag}\left(a^{t} b\right) \equiv \operatorname{diag}\left(c^{t} d\right) \equiv 0 \bmod 2 l$.

If we drop the last condition we get the principal congruence subgroup $\Gamma_{g}(l)$ of level $l$. Let $\Gamma$ be any of these groups; then for every non negative integer $k$ we consider the vector space $[\Gamma, k]$ over $C$ of Siegel modular forms of weight $k$, i.e. holomorphic functions $\psi$ on $\boldsymbol{H}_{\boldsymbol{g}}$ satisfying the functional equation

$$
\psi(\sigma \cdot \tau)=\operatorname{det}(c \tau+d){ }^{k} \psi(\tau)
$$

for every $\sigma$ in $\Gamma$. The graded ring

$$
A(\Gamma)=\bigoplus_{0 \leq k \leq \infty}[\Gamma, k]
$$

is an integrally closed domain of finite type over $C$. We recall from the introduction that

$$
\Theta_{g}(2) \subseteq A\left(\Gamma_{g}(4,8)\right)
$$

and the morphism 


$$
\alpha: \operatorname{Proj} A\left(\Gamma_{g}(4,8)\right) \longrightarrow \operatorname{Proj} \Theta_{g}(2)
$$

is bijective.

We want to analyze the maps

$$
\alpha_{2}: \operatorname{Proj} A\left(\Gamma_{g}(2)\right) \longrightarrow \operatorname{Proj} B_{g}(2)
$$

and

$$
\alpha_{1}: \operatorname{Proj} A\left(\Gamma_{g}\right) \longrightarrow \operatorname{Proj} B_{g} .
$$

We shall apply the same method of Igusa in [8], therefore we need to recall the structure of the holomorphic local ring of Proj $A(\Gamma)$ at a point $P$. We take as $P$ the unique special point defined by a sequence in $\boldsymbol{H}_{g}$ such that the imaginary part of a general terms tends to $\infty$. Moreover we put $\alpha_{i}(P)=Q, i=1,2$.

We know that holomorphic local ring at $P$ consists of all convergent series of the form

$$
f=\sum_{S} \operatorname{const} \Phi_{S}
$$

in which $S$ runs over a complete set of representatives of $S L(g, Z)(l)$ equivalence classes in the set of even semi-positive definite matrices, $l=1$ and 2 . Here we denoted by $S L(g, Z)(l)$ the normal subgroup of $S L(g, Z)$ that is the kernel of the morphism

$$
\phi: S L(g, Z) \longrightarrow S L(g, Z / l Z) .
$$

Moreover we have

$$
\Phi_{S}=\sum_{u \in S L(g, Z)(l)} e\left(\frac{1}{2 l} \operatorname{tr}\left(S u \tau^{t} u\right)\right)
$$

really the summation is over the set of distinct ${ }^{t} u S u$.

From now on, to avoid confusion, we develop only one computation since they are similar, therefore we shall consider only the map

$$
\alpha_{1}: \operatorname{Proj} A\left(\Gamma_{g}\right) \longrightarrow \operatorname{Proj} B_{g} .
$$

Let $\tau_{i j}$ denote the $(i, j)$-th coefficient of a point $\tau$ in $\boldsymbol{H}_{g}$; then we define the following $g(g+1) / 2$ analytically independent holomorphic functions on $H_{g}$

$$
q_{i j}(\tau)=\mathbf{e}\left(\tau_{i j}\right) \quad 1 \leq i \leq j \leq g .
$$

Let $O_{P}$ and $O_{Q}$ be the local ring at $P$ and $Q=\alpha_{1}(P)$; it is a well known 
fact that $O_{P}$ and the holomorphic local ring have the same completion. Let us denote by $C \llbracket q \rrbracket$ the ring of formal power series in $q_{i j}$ then we have

$$
O_{Q} \subseteq O_{P} \subseteq C \llbracket q \rrbracket
$$

and passing to the completion we have

$$
O_{Q}^{*} \subseteq O_{P}^{*} \subseteq C \llbracket q \rrbracket,
$$

since even Proj $B_{g}$ is an analytically irreducible variety. Let us assume $g \geq 47$; then we denote by $P_{g}$ the matrix obtained by $P_{47}$ adding $g-47$ rows and columns of zero then we have;

Theorem 1. Suppose that $g \geq 47$. Then $\Phi_{P_{g}}$ is in $O_{P}^{*}$ but not in $O_{Q}^{*}$. Therefore the bijective morphism $\alpha_{1}$ is not an isomorphism.

Proof. As pointed in [8] we have that an element of $O_{Q}$ is of the form

$$
f=\sum \text { const. } \Phi_{S}
$$

in which $4 S=\xi^{t} \xi+\eta^{t} \eta+\cdots$ for some $\xi$, $\eta$ in $Z^{g}$ and an element of $O_{Q}^{*}$ also has the same form. This clearly is not the case for $\Phi_{P_{g}}$ by Lemma 1 .

Clearly we have;

Theorem 2. Suppose that $g \geq 22$. Then $\alpha_{2}$ is not an isomorphism.

Consequently we have

THEOREM 3. If $g \geq 47$ (or 22) there exists a modular form of arbitrarily high weight relative to $\Gamma_{g}\left(\right.$ or $\left.\Gamma_{g}(2)\right)$ which can not be expressed as a polynomial in the Thetanullwerte with half integral characteristics.

$\S 3$.

In this section we shall recall some basic facts about Thetanullwerte and Hecke operators.

For any associative ring $R$ with unity we shall denote by $M_{p, q}(R)$ the $R$-module of $p \times q$ matrices with coefficients in $R$. We shall write $M_{p}(R)$ for $M_{p, p}(R)$. If $A$ is in $M_{p}(R)$ we shall denote by $\operatorname{diag}_{k}(A)$ the element of $M_{p, k}(R)$ defined by $\operatorname{diag}_{k}(A)_{i j}=A_{i i}$ for all $i$ and $j$, we shall omit $k$ if it is 1 .

We know that $\Gamma_{g}$ acts on the set $M_{2 g, p}(\boldsymbol{Q} / Z)$ via 


$$
\sigma \cdot M={ }^{t} \sigma^{-1} M+1 / 2\left(\begin{array}{l}
\operatorname{diag}_{p}\left(c^{t} d\right) \\
\operatorname{diag}_{p}\left(a^{t} b\right)
\end{array}\right)
$$

for $M$ in $M_{2 g, p}(\boldsymbol{Q})$, more explicitly if we put

$$
M=\left(\begin{array}{l}
M^{\prime} \\
M^{\prime \prime}
\end{array}\right)
$$

with $M^{\prime}, M^{\prime \prime}$ in $M_{g, p}(\boldsymbol{Q})$, we have

$$
\sigma \cdot M=\left(\begin{array}{rr}
d & -c \\
-b & a
\end{array}\right)\left(\begin{array}{l}
M^{\prime} \\
M^{\prime \prime}
\end{array}\right)+1 / 2\left(\begin{array}{l}
\operatorname{diag}_{p}\left(c^{t} d\right) \\
\operatorname{diag}_{p}\left(a^{t} b\right)
\end{array}\right)
$$

If $M$ is half integral, i.e., $2 M \equiv 0 \bmod 1$, we can describe the orbits of the $\Gamma_{g}$ action. Let $m, m_{1}, m_{2}, m_{3}$ be half integral vectors in $\boldsymbol{Q}^{2 g}$, we put

$$
e(m)=e\left(2^{t} m^{\prime} m^{\prime \prime}\right), e\left(m_{1}, m_{2}, m_{3}\right)=e\left(m_{1}\right) e\left(m_{2}\right) e\left(m_{3}\right) e\left(m_{1}+m_{2}+m_{3}\right) .
$$

Let $M=\left(m_{1}, m_{2}, \cdots m_{p}\right)$ be a sequence of half integral vectors in $\boldsymbol{Q}^{2 g}$; we say that it is essentially independent if for any choice of $1 \leq i_{i}<i_{2}$ $<i_{2 k} \leq p, k \geq 1$ we have

$$
m_{i_{1}}+m_{i_{2}}+\cdots+m_{i_{2 k}} \not \equiv 0 \bmod 1 .
$$

With these notations we have: two sequence of half integral vectors in $\boldsymbol{Q}^{2 g} \quad M=\left(m_{1}, m_{2}, \cdots m_{p}\right)$ and $N=\left(n_{1}, n_{2}, \cdots, n_{p}\right)$ are conjugate under the action of $\Gamma_{g}$ if and only if essentially independent subsequences correspond to each other, and further

$$
e\left(m_{i}\right)=e\left(n_{i}\right) \text { and } e\left(m_{i}, m_{j}, m_{k}\right)=e\left(n_{i}, n_{j}, n_{k}\right)
$$

for all $1 \leq i \leq p$ and $1<i<j<k \leq p$.

We take $M=\left(m_{1} \cdots m_{p}\right)$ arbitrarily from $M_{2 g, p}(\boldsymbol{Q})$ and put

$$
P(M)=\vartheta_{m_{1}} \cdots \vartheta_{m_{p}} .
$$

For such monomials in the Thetanullwerte we recall the transformation formula

$$
P(\sigma \cdot M)(\sigma \cdot \tau)=k(\sigma)^{p} \operatorname{det}(c \tau+d)^{p / 2} e\left(\Phi_{M}(\sigma)\right) P(M)(\tau)
$$

for all $\sigma$ in $\Gamma_{g}$.

We know that $k(\sigma)^{2}$ depends only on $\sigma$ and

$$
k(\sigma)^{4}=e\left(\frac{1}{2} \operatorname{tr}\left({ }^{t} b c\right)\right),
$$


while

$$
\begin{aligned}
\Phi_{M}(\sigma)=-1 / 2 \operatorname{tr}\left({ }^{t} M^{\prime}{ }^{t} b d M^{\prime}+{ }^{t} M^{\prime \prime}{ }^{t} a c M^{\prime \prime}-2{ }^{t} M^{\prime}{ }^{t} b c M^{\prime \prime}\right. \\
\\
\left.-{ }^{t} \operatorname{diag}_{p}\left(a^{t} b\right)\left(d M^{\prime}-c M^{\prime \prime}\right)\right)
\end{aligned}
$$

Finally for $k$ even positive, we recall that

$$
B_{g}(2)_{k}=\left[\Gamma_{g}(2), k\right] \cap B_{g}(2)
$$

is the $C$-span of all monomials $P(M)$ with $M$ in $M_{2 g, 2 k}(\boldsymbol{Q})$ half integral satisfying the following congruences;

$$
4 M^{t} M \equiv 0 \bmod 2 \text { and } \operatorname{diag}\left(M^{t} M\right) \equiv 0 \bmod 1, \text { cf. [6] }
$$

and the elements of $\left[\Gamma_{g}, k\right] \cap B_{g}=B_{g, k}$ are symmetrizations of the elements of $B_{g}(2)_{k}$, i.e., they are linear combinations of functions of the form

$$
\begin{aligned}
f_{M}(\tau) & =\sum_{\sigma \in S p(g, \boldsymbol{Z}) / \Gamma g^{(2)}} \operatorname{det}(c \tau+d)^{-k} P(M)(\sigma \cdot \tau) \\
& =\sum_{\sigma \in S p(g, Z) / \Gamma g^{(2)}} k(\sigma)^{2 k} \mathbf{e}\left(\Phi_{\sigma-1 \cdot M}(\sigma)\right) P\left(\sigma^{-1} \cdot M\right)(\tau) .
\end{aligned}
$$

Since we have

$$
\vartheta_{m+n}(\tau)=\mathbf{e}\left({ }^{t} m^{\prime} n^{\prime}\right) \vartheta_{m}(\tau)
$$

for every $n$ in $Z^{2 g}$, we can assume the coefficients of $M$ equal to 0 or $1 / 2$ and if we denote by $\sigma^{-1} \circ M$ the unique element congruent to $\sigma^{-1} \cdot M$ $\bmod 1$ with 0 and $1 / 2$ as coefficients, then we can write

$$
\begin{aligned}
f_{M}(\tau)= & \sum_{\sigma \in S p(g, Z) / \Gamma_{g}(2)} k(\sigma)^{2 k} e\left(\Phi_{\sigma-1 \cdot M}(\sigma)\right) \mathbf{e}\left(\operatorname{tr}\left({ }^{t}\left(\sigma^{-1} \circ M\right)^{\prime}\left(\sigma^{-1} \cdot M-\sigma^{-1} \circ M\right)^{\prime \prime}\right)\right) \\
& \times P\left(\sigma^{-1} \circ M\right)(\tau) .
\end{aligned}
$$

With these notations we have the following

Lemma. Assume that $k>g+1$ is even. Then $B_{g, k} \neq 0$.

Proof. First let us recall that any $f$ in $A\left(\Gamma_{g}\right)$ has a Fourier expansion, namely

$$
f(\tau)=\sum_{T} a(T) e\left(\frac{1}{2} \operatorname{tr}\left(T_{\tau}\right)\right)
$$

We are summing over the set of even semipositive definite matrices. Now let $M_{1}$ be such that $2 M_{1}=0$ and 


$$
\begin{aligned}
& 2 M_{1}^{\prime \prime}=(e_{1}, e_{2}, \cdots, e_{g}, v_{g}, e_{1}, e_{2}, \cdots, e_{g}, v_{g}, \underbrace{0 \cdots 0}_{\begin{array}{c}
2 k-2 g-2 \\
\text { times }
\end{array}}) \\
& =\left[\begin{array}{ccccccccccccc}
1 & 0 & . & 0 & 1 & 1 & 0 & \cdot & 0 & 1 & 0 & . & 0 \\
0 & 1 & . & 0 & 1 & 0 & 1 & . & 0 & 1 & 0 & . & 0 \\
. & . & . & . & . & . & . & . & . & . & . & . & . \\
. & . & . & . & . & . & . & . & . & . & . & . & . \\
0 & . & 0 & 1 & 1 & 0 & . & 0 & 1 & 1 & 0 & . & 0
\end{array}\right]
\end{aligned}
$$

Here we denoted by $v_{g}$ the sum of the $g$ unit vectors.

We shall compute the Fourier coefficient $a(0)$ of $f_{M_{1}}(\tau)$. We have

$$
a(0)=\sum k(\sigma)^{2 k} e\left(\Phi_{\sigma-1 \cdot M_{1}}(\sigma)\right)
$$

where the summation is over $\sigma \in S p(g, Z) / \Gamma_{g}(2)$ such that

$$
\left(\sigma^{-1} \circ M_{1}\right)^{\prime}=0 \text {. }
$$

First we analyze the condition $\left(\sigma^{-1} \circ M_{1}\right)^{\prime}=0$. From (11) we get

$$
\begin{aligned}
\left(\sigma^{-1} \circ M_{1}\right)^{\prime} & \equiv{ }^{t} c M^{\prime \prime}-1 / 2 \operatorname{diag}_{2 k}\left({ }^{t} c a\right) \\
& \equiv\left({ }^{t} c,{ }^{t} c v_{g},{ }^{t} c,{ }^{t} c v_{g}, 0\right)-1 / 2 \operatorname{diag}_{2 k}\left({ }^{t} c a\right) \bmod 1
\end{aligned}
$$

Now the condition (21) implies $\operatorname{diag}_{2 k}\left({ }^{t} c a\right) \equiv 0 \bmod 2$ and $c \equiv 0 \bmod 2$; therefore if we put

$$
\Gamma_{g, 0}(2)=\left\{\sigma \in \Gamma_{g} \mid c \equiv 0 \bmod 2\right\}
$$

we get

$$
a(0)=\sum_{\sigma \in \Gamma_{g}, 0(2) / \Gamma_{g^{(2)}}} k(\sigma)^{2 k} e\left(\phi_{\sigma-1 \cdot M_{1}}(\sigma)\right)
$$

From (14) and (15) we get $k(\sigma)^{2 k}=1$ and

$$
\begin{aligned}
\phi_{\sigma-1 \cdot M_{1}}(\sigma) \equiv & -1 / 2 \operatorname{tr}\left({ }^{t}\left({ }^{t} M_{1}^{\prime \prime} d-1 / 2 \operatorname{diag}_{2 k}\left({ }^{t} d b\right)\right){ }^{t} a c\left({ }^{t} d M_{1}^{\prime \prime}-1 / 2 \operatorname{diag}_{2 k}\left({ }^{t} d b\right)\right)\right) \\
\equiv & -1 / 2 \operatorname{tr}\left({ }^{t} M_{1}^{\prime \prime} d^{t} a c^{t} d M_{1}^{\prime \prime}-1 / 2{ }^{t} \operatorname{diag}_{2 k}\left({ }^{t} d b\right){ }^{t} a c^{t} d M_{1}^{\prime \prime}\right. \\
& \left.-1 / 2{ }^{t} M_{1}^{\prime \prime} d^{t} a c \operatorname{diag}_{2 k}\left({ }^{t} d b\right)\right) \bmod 1
\end{aligned}
$$

Since the vectors of $M_{1}$ are essentially dependent and

$$
\operatorname{diag}_{2 k}\left({ }^{t} d b\right)^{t} \operatorname{diag}_{2 k}\left({ }^{t} d b\right) \equiv 0 \bmod 4,
$$

we have

$$
\phi_{\sigma-1, M_{1}}(\sigma) \equiv-\operatorname{tr}\left(1 / 2 d^{t} a c^{t} d M_{1}^{\prime \prime}{ }^{t} M_{1}^{\prime \prime}\right) \bmod 1,
$$

but $1 / 2 d^{t} a c^{t} d$ is an integral symmetric matrix and $M_{1}^{\prime \prime}$ satisfies (16), therefore $\phi_{o-1 . M_{1}}(\sigma)=1$ for any $\sigma$ in $\Gamma_{g, 0}(2)$ and we have 


$$
a(0) \neq 0 \text {. }
$$

We recall some basic facts about Hecke operators. We know that for every prime $p$ the Hecke operator $T(p)$ on the space $\left[\Gamma_{g}, k\right]$ defined by

(23) $\quad T(p) \cdot f=\left.f\right|_{T(p)}(\tau)$

$$
=p^{g k-(g(g+1)) / 2} \sum_{\sigma \in \Gamma_{g} / \Gamma_{g}\left(\begin{array}{cc}
1 \\
0 \\
0 & 0 \\
p 1_{g} g
\end{array}\right) \Gamma_{g}} \operatorname{det}(c \tau+d)^{-k} f\left((a \tau+b)(c \tau+d)^{-1}\right)
$$

is well defined and maps $\left[\Gamma_{g}, k\right]$ to itself.

Moreover if we restrict to modular forms that can be expressed as polynomials in the Thetanullwerte we learned from Böcherer that the Hecke operator $T(p)$ maps $\left[\Gamma_{g}, k\right] \cap \Theta_{g}(r)$ in $\left[\Gamma_{g}, k\right] \cap \Theta_{g}(p r)$. Therefore the graded ring $\Theta_{g}(r)$ are not stable under the action of Hecke operators. Nevertheless we obtain that $C_{g}(n)$ is stable under Hecke operators $T(p)$ when $p$ divides $n$. Let us recall that the Eisenstein series

$$
E_{k}(\tau)=\sum_{\sigma \in \Gamma_{\boldsymbol{g}}, 0 / \Gamma_{g}} \operatorname{det}(c \tau+d)^{-k} \quad k>g+1 \text { even }
$$

belongs to $\left[\Gamma_{g}, k\right]$ and it is an eigenform for all Hecke operator. Here we denoted by $\Gamma_{g, 0}$ the subgroup of $\Gamma_{g}$ defined by $c=0$. Moreover it is, up to a constant, the unique element of $\left[\Gamma_{g}, k\right]$ that is an eigenform for $T(p)$ for at least one prime $p$ and has the Fourier coefficient $a(0)$ different from $0 \mathrm{cf}$ [4] and [5]. Moreover let us recall from [5] the following.

TheOREm 4. Let $V$ be a subgroup of $\left[\Gamma_{n}, k\right]$ which is stable under the action of a certain subset of Hecke operators, then $V$ has a basis of simultaneous eigenfunctions for those operators.

Theorem 5. Let $V$ be as and assume that in $V$ there is a modular form with the Fourier coefficient a(0) different from zero, then there exists an eigenform with the same property.

As immediate consequence of the above Theorems we have

Corollary. The Eisenstein series $E_{k}(\tau)(k>g+1)$ are contained in the graded ring $C_{g}(n)$ for all positive $n$.

At this point we can do the same reasoning of Böcherer in [1] to get the final results. We repeat it sketchly. First, let us recall two elementary properties of Thetanullwerte. Assume $g=r+s$. Then for any $A$ in $M_{g, p}(\boldsymbol{Q})$ we shall denote by $A^{*}$ the matrix obtained from $A$ taking the 
first $r$ rows and $A^{* *}$ the matrix obtained from the remaining $s$ rows. For any $M$ in $M_{2 g, p}(\boldsymbol{Q})$ we shall write, by an abuse of notation,

$$
M^{*}=\left(\begin{array}{c}
M^{\prime *} \\
M^{\prime *}
\end{array}\right), \quad M^{* *}=\left(\begin{array}{c}
M^{\prime * *} \\
M^{\prime * *}
\end{array}\right) .
$$

Let $\tau^{\prime}, \tau^{\prime \prime}$ be arbitrary point of $\boldsymbol{H}_{r}$ and $\boldsymbol{H}_{s}$ then it is immediate to verify

$$
P(M)\left(\begin{array}{ll}
\tau^{\prime} & 0 \\
0 & \tau^{\prime \prime}
\end{array}\right)=P\left(M^{*}\right)\left(\tau^{\prime}\right) P\left(M^{* *}\right)\left(\tau^{\prime \prime}\right)
$$

moreover for all $\tau$ in $\boldsymbol{H}_{g}$ we have

$$
\overline{P(M)(-\bar{\tau})}=P(M)(\tau) .
$$

Using the two above properties of Thetanullwerte and the result of the previous corollary we can apply Böcherer's method. From these we have

Theorem 6. Assume that $k>2 g$ is even. Then any $f$ in $\left[\Gamma_{g}, k\right]$ can bo expressed as a homogeneous polynomial belonging to $C_{g}(n)$.

For details we refer to [1] and [2].

\section{REFERENCES}

[1] S. Böcherer, Über die Fourier-Jacobi-Entwicklung Siegelscher Eisensteinreihen, Math. Z., 183 (1983), 21-46.

[2] - Siegel modular forms and theta series, Proc. Symp. P. Math., 49 (1989).

[ 3 ] Conway Sloane, Sphere Packings, Lattices and Groups, Grund Math. Wiss. 290, Springer Verlag, Berlin-Heidelberg-New York (1988).

[4] J. Elstrodt, Eine Charakterisierung der Eisenstein-Reihe zur Siegelschen Modulgruppe,

[ 5 ] E. Freitag, Siegelsche Modulfunktionen, Grund. Math. Wiss. 254, Springer Verlag, Berlin-Heidelberg-New York (1983).

[6 ] J. Igusa, On the graded ring of theta-constants, Amer. J. Math.: I, 86 (1964), 219-246; II, 88 (1966), 221-236.

[ 7 ] —-, On Siegel modular forms of genus two II, Amer. J. Math., 86 (1964), 392-412.

[8] - On the variety associated with the ring of thetanullwerte, Amer. J. Math., 103 (1981), 377-398.

[9] - A desingularization problem in the theory of Siegel modular functions, Math. Ann., 168 (1967), 228-260.

[10] — - On the Nullwerte of jacobians of odd theta functions, Symp. Math., XXIV

[11] R. Salvati Manni, On the not integrally closed subrings of the ring of the Thetanullwerte II, J. Reine angew. Math., 372 (1986), 64-70.

[12] — - Thetanullwerte and stable modular forms, Amer. J. Math., 111 (1989), 435455.

[13] S. Tsuyumine, On Siegel modular forms of degree three, Amer. J. Math., 108 (1986), 755-862. 
Dipartimento di Matematica Università «La Sapienza» 00185 Roma

Italy 